\title{
Clinicopathological characteristics of extremely young Korean multiple myeloma patients: therapeutic implications
}

Junghoon Shin ${ }^{1,}$, Youngil Koh ${ }^{1,}$, Jeonghwan Youk ${ }^{1}$, Miso Kim ${ }^{1}$, Byung Soo Kim², Chul Won Choi ${ }^{2}$, Hwa Jung Sung ${ }^{2}$, Yong Park ${ }^{2}$, Sung-Soo Yoon ${ }^{1}$, and Inho Kim ${ }^{1}$

${ }^{1}$ Department of Internal Medicine, Seoul National University Hospital, Cancer Research Institute, Seoul National University College of Medicine, Seoul; ${ }^{2}$ Department of Internal Medicine, Korea University College of Medicine, Seoul, Korea

Received: August 3, 2016

Revised : October 18, 2016

Accepted: February 4, 2017

\section{Correspondence to}

Inho Kim, M.D.

Department of Internal Medicine, Seoul National University Hospital, 101 Daehak-ro, Jongnogu, Seoul 03080, Korea

Tel: +82-2-2072-0834

Fax: +82-2-764-2199

E-mail: ihkimmd@snu.ac.kr

*These authors contributed equally to this work.
Background/Aims: Although multiple myeloma (MM) is typically a disease of the elderly, a certain subset of extremely young patients exists. It is necessary to establish clinicopathological characteristics for this population.

Methods: We reviewed the medical records of MM patients whose age was 40 years or younger at diagnosis.

Results: A total of 32 patients were analyzed (male to female ratio 19:13, median age 37 years). According to International Staging System, 29\%, 48\%, and 16\% were in stage I, II, and III, respectively. Light chain myeloma accounted for $30 \%$. Clinically significant anemia, hypercalcemia, azotemia, and hypoalbuminemia were present in $29 \%, 28 \%, 13 \%$, and $28 \%$, respectively. Three or more lytic bone lesions were detected in $45 \%$ of the patients, whereas $13 \%$ had no lytic bone lesions. Regarding treatment, $79 \%$ of patients received autologous hematopoietic stem cell transplantation. After a median follow-up duration of 64 months, the 1-, 3-, and 5 -year overall survival (OS) rates were $84 \%, 62 \%$, and $54 \%$, respectively. The median OS was 61 months for the entire cohort.

Conclusions: In our study, MM patients aged 40 years or younger at diagnosis showed no superior survival compared to those of the moderately elderly patients based on historical data.

Keywords: Multiple myeloma; Young adult; Hematopoietic stem cell transplantation; Treatment outcome

\section{INTRODUCTION}

Multiple myeloma (MM) is a disease of the elderly; it shows a rapidly increasing incidence with increased age. The peak age-specific incidence has been observed in people older than 80 years of age, where it reaches approximately 40 per 100,000 person-years [1]. Although $\mathrm{MM}$ is rare in young people, patients diagnosed before the age of 50 years account for $10 \%$ of the overall incidence, and approximately $2 \%$ are diagnosed before 40 years of age [2]. Various reports have examined the different clinical and laboratory features and treatment outcome for such young MM patients [2-8]. Although some of these data are debatable, those patients generally seem to be at lower International Staging System (ISS) and Durie-Salmon Staging System (DSS) stages at diagnosis, more frequently present with light-chain myeloma, have a lower proportion of measurable $\mathrm{M}$ protein and bone marrow (BM) plasmacytosis, and have a lower frequency of related tissue injuries, including 
hypercalcemia, azotemia, and anemia, compared with conventional elderly patients $[3,4]$.

The overall survival (OS) of MM shows remarkable variation, ranging from a few months to more than 10 years. Because of this prognostic diversity, the necessity to stratify patients into divided risk groups has emerged. The prognosis of MM patients has been associated with several parameters of prognostic significance [2]. Those include the initial performance status, the ISS and DSS stage at diagnosis, indicators of related tissue injuries, plasma cell labeling index, and certain chromosomal aberrations $[9,10]$. However, the role of age as an independent prognostic factor, especially when the life expectancy of the corresponding general population is taken into account, has been part of debate. In a previous study comparing patients younger than 50 years at diagnosis with those aged 50 years or older, the younger group showed better survival in terms of observed survival rates; however, that group appeared to have a significantly worse outcome when the mortality was standardized with the expected death rate of the corresponding general population [2]. A more recently reported retrospective study also suggested that MM patients diagnosed before 40 years of age do not show significant differences in OS after autologous hematopoietic stem cell transplantation (aHSCT) compared to those aged 41 to 65 years [8]. In contrast, some large studies suggested that old age is associated with short survival $[4,11]$. Therefore, the importance of age as an independent prognostic factor in MM is still unclear.

Although evidence from several studies has implied that Asian MM patients could show clinical behavior and adverse drug reaction patterns that differ from those of Western patients [12-14], studies on the presenting features and long-term outcomes of young Asian MM patients have rarely been conducted. In this study, we analyzed the clinicopathological characteristics, the outcomes of several treatment approaches, and the prognosis of Korean MM patients diagnosed at age 40 years or younger.

\section{METHODS}

\section{Eligible patients and data collection}

Four institutes in South Korea participated in this study.
Patients diagnosed with $\mathrm{MM}$ at the age of 40 years or younger from January 2000 to February 2015 were retrieved through a retrospective search of electronic medical records (EMRs). The Institutional Review Board of Seoul National University Hospital (SNUH) approved the study protocol (IRB approval number H-1305-629491). All procedures were in accordance with the ethical standards of the institutional research committee and with the Declaration of Helsinki revised in the 2013 World Medical Association. The International Myeloma Working Group's diagnostic and response criteria on the date of diagnosis and the response evaluation for each patient were used to enroll the patient and analyze the data regarding the treatment response. Monoclonal gammopathy of unknown significance, smoldering myeloma, amyloidosis, and POEMS (polyneuropathy, organomegaly, endocrinopathy, monoclonal gammopathy, and skin changes) syndrome were excluded.

The stage at diagnosis was classified according to both ISS, described by International Myeloma Working Group in 2005, and DSS, first described in 1975. The number and location of osteolytic lesions were analyzed by reviewing the skeletal survey images from X-rays and magnetic resonance imaging, if available. BM examination results were collected, including the proportion of plasma cell infiltration, conventional cytogenetics, and fluorescence in situ hybridization (FISH) for chromosomal aberrations with prognostic implications [15]. The starting date, regimen, maximal response, and the date of progression or death for first-line treatment, the bortezomib-containing regimen, and aHSCT were also reviewed. The causes of death were categorized into MM progression, infection, thrombosis, or others.

\section{Statistical analysis}

Progression-free survival (PFS) was defined as the interval from the beginning of each treatment to progression or death from any cause, whichever occurred first. OS was defined as the interval from diagnosis to death from any cause. Patients whose date of progression or death was uncertain or who were progression-free or alive on the date of the data collection were censored in the survival analysis using the last date they were known to be progression-free and alive. The PFS after first-line treatment, aHSCT and bortezomib-containing regimen and OS were calculated for each evaluable patient. A survival 
Table 1. Baseline characteristics of the patients

\begin{tabular}{|c|c|}
\hline Characteristic & Frequency/Total $^{\mathrm{a}}(\%)$ \\
\hline \multicolumn{2}{|l|}{ Sex } \\
\hline Male & $19 / 32(59)$ \\
\hline Female & $13 / 32(41)$ \\
\hline \multicolumn{2}{|l|}{ ECOG } \\
\hline o & $4 / 31(13)$ \\
\hline 1 & $13 / 31(42)$ \\
\hline 2 & $8 / 31(26)$ \\
\hline 3 & $5 / 31(16)$ \\
\hline 4 & $1 / 31(3)$ \\
\hline \multicolumn{2}{|l|}{ ISS } \\
\hline I & $10 / 31(32)$ \\
\hline II & $15 / 31(48)$ \\
\hline III & $6 / 31(19)$ \\
\hline \multicolumn{2}{|l|}{ DSS } \\
\hline I & $5 / 31(16)$ \\
\hline II & $8 / 31(26)$ \\
\hline III & $18 / 31(58)$ \\
\hline Subtype A & $27 / 31(87)$ \\
\hline Subtype B & $4 / 31(13)$ \\
\hline \multicolumn{2}{|l|}{ Subtype } \\
\hline \multicolumn{2}{|l|}{ Light chain } \\
\hline$\kappa$ & $4 / 30(13)$ \\
\hline$\lambda$ & $5 / 30(17)$ \\
\hline \multicolumn{2}{|l|}{ Whole immunoglobulin } \\
\hline Immunoglobulin G & $14 / 30(47)$ \\
\hline Immunoglobulin A & $5 / 30(17)$ \\
\hline Immunoglobulin D & $2 / 30(7)$ \\
\hline$\kappa$ & $13 / 30(43)$ \\
\hline$\lambda$ & $8 / 30(27)$ \\
\hline Hemoglobin < $10 \mathrm{~g} / \mathrm{dL}$ & $9 / 31(29)$ \\
\hline Platelet $<130 \times 10^{3} / \mu \mathrm{L}$ & $7 / 31(23)$ \\
\hline Calcium $^{\mathrm{b}} \geq 10 \mathrm{mg} / \mathrm{dL}$ & $9 / 32(28)$ \\
\hline Creatinine $\geq 2 \mathrm{mg} / \mathrm{dL}$ & $4 / 32(13)$ \\
\hline Albumin <3.5 g/dL & $9 / 32(28)$ \\
\hline $\mathrm{B} 2 \mathrm{MG} \geq 3.5 \mathrm{mg} / \mathrm{dL}$ & $14 / 29(48)$ \\
\hline LDH above normal & $10 / 27(37)$ \\
\hline \multicolumn{2}{|l|}{ Lytic bone lesions } \\
\hline 0 & $4 / 31(13)$ \\
\hline $1-2$ & $13 / 31(42)$ \\
\hline$\geq 3$ & $14 / 31(45)$ \\
\hline \multicolumn{2}{|l|}{ BM plasma cells, $\%$} \\
\hline $10-30$ & $15 / 27(56)$ \\
\hline$\geq 30$ & $7 / 27(26)$ \\
\hline Biopsy-proven plasmacytoma & $20 / 31(65)$ \\
\hline
\end{tabular}

ECOG, Eastern Cooperative Oncology Group; ISS, International Staging System; DSS, Durie-Salmon Staging System; B2MG, $\beta-2$ microglobulin; $\mathrm{LDH}$, lactate dehydrogenase; $\mathrm{BM}$, bone marrow.

Total indicates the number of patients with sufficient data for categorization.

${ }^{\mathrm{b}}$ Corrected calcium level, calculated from measured calcium $+0.8 \times(4-$ albumin $)$. curve was constructed using the Kaplan-Meier method for the entire cohort based on the observed mortality and used to estimate the median PFS and OS, 1-, 3-, and 5-year PFS and OS rates. To compare survival between two groups, a log-rank test was used. The results were considered statistically significant at two-sided $p<0.05$. STATA statistical software version 12.0 (StataCorp LP, College Station, TX, USA) was used for computation.

\section{RESULTS}

\section{Baseline characteristics}

A total of 49 patients were retrieved via computer-based searching of the EMRs of four institutions. Among those patients, 13 and four were excluded from the analysis because of the erroneous input of the diagnosis and insufficient medical records, respectively. Of the 32 patients who were included in the analysis, 25 were treated at SNUH, four at Korea University Anam Hospital, two at Korea University Ansan Hospital, and one at Korea University Guro Hospital.

Patient characteristics are summarized in Table 1. The median age at diagnosis for all 32 patients was 37 years (range, 17 to 40). Of those, four patients (two males and two females) were younger than 30 years at diagnosis. The age distribution at diagnosis was similar irrespective of gender. Twenty patients were diagnosed before 2010 while the remaining patients were diagnosed in 2010 or thereafter. The most common subtype of Mprotein was immunoglobulin G (IgG), followed by IgA and IgD. Light chain myeloma accounted for $30 \%$ of patients. Data regarding specific locations of bony lesions were available for 20 patients treated at SNUH. The most commonly involved bones were the vertebrae and skull, accounting for $60 \%$ and $55 \%$ of the patients with bone involvement, respectively; these were followed by the ribs, pelvis, femur, humerus, and scapulae, accounting for $40 \%, 30 \%, 30 \%, 20 \%$, and $10 \%$, respectively. Karyotypes of the BM cells determined by the conventional G-banding technique were available for 23 patients. The chromosomal abnormality was observed in $30 \%$ of the patients according to conventional karyotyping. When considering both of the conventional karyotyping and FISH, the proportion grew up to $59 \%$. The prevalence of each adverse genetic lesion detected using FISH is 
summarized in Table 2. The chromosomal aberrations included in the table were selected based on previous reports showing their significant association with shorter PFS and OS $[10,16]$. Regarding initial manifestation, 20 (65\%) out of the 31 patients evaluated for detectable tumors using an imaging study were found to have plasmacytomas which were pathologically confirmed. Those patients showed a tendency toward a low probability of $\mathrm{BM}$ plasmacytosis (BM plasma cell fraction $\geq 10 \%$; $p=$ 0.137 with a Fisher exact test).

Table 2. Frequency of adverse chromosomal alterations

\begin{tabular}{|c|c|c|}
\hline \multicolumn{2}{|c|}{ High-risk FISH features } & \multirow[b]{2}{*}{$\begin{array}{c}\text { Frequency/ } \\
\text { Total }^{\mathrm{a}}(\%)\end{array}$} \\
\hline $\begin{array}{c}\text { Chromosomal } \\
\text { alteration }\end{array}$ & Genetic involvement & \\
\hline $\operatorname{del}(17 p)$ & p53 deletion & $1 / 9(11)$ \\
\hline $\mathrm{t}(4 ; 14)$ & IGH/FGFR3 rearrangement & $1 / 10(10)$ \\
\hline$t(14 ; 16)$ & IGH/MAF rearrangement & $\mathrm{o} / 11(0)$ \\
\hline$t(14 ; 20)$ & & $\mathrm{o} / 6(0)$ \\
\hline 1q gain & & $4 / 15(0)$ \\
\hline $\operatorname{del}(13 q)$ & rbı deletion & $4 / 17(24)$ \\
\hline $\operatorname{del}(9)$ & p16 (CDKN2A) deletion & $1 / 16(6)$ \\
\hline
\end{tabular}

FISH, fluorescence in situ hybridization.

${ }^{\text {aT }}$ Total indicates the number of patients tested for each chromosomal alteration.

\section{Response to treatment}

Regarding treatment, the first-line chemotherapeutic regimens administered are summarized in Table 3. Two patients were transferred to other institutions right after diagnosis, leaving no records regarding treatment. Either vincristine/doxorubicin/dexamethasone (VAD) alone or two cycles of VAD subsequently followed by bortezomib/thalidomide/dexamethasone (VTD) was the most frequently used regimen, followed by thalidomide/dexamethasone (TD). Among the patients whose response to the first-line treatment could be evaluated, either a complete response (CR) or a very good partial response (VGPR) was achieved in 14 patients (48\%) after first-line chemotherapy with or without subsequent aHSCT. Of these patients, five were not eligible for aHSCT because of early progression before stem cell collection $(n=2)$, the patient's refusal $(n=2)$, and suicide immediately after the initial response evaluation $(n=1)$. Regarding the 26 patients with relevant data, 18 (69\%) eventually experienced progressive disease (PD). The median PFS after first-line treatment was 16 months ( $95 \%$ confidence interval [CI], 9 to 30) with 1-, 3-, and 5-year PFS rates of $65 \%$ (95\% CI, $42 \%$ to $81 \%$ ), $27 \%$ (95\% CI, $10 \%$ to $48 \%$ ), and $14 \%$ (95\% CI, 3\% to 34\%), respectively (Fig. 1A). The patients who received aHSCT as a first-line treatment showed a tendency toward improved PFS compared with the patients who did not (median 17 months vs. 3 months, $p=$ 0.101) (Fig. 1B).

Table 3. First-line therapeutic regimens

\begin{tabular}{lccc}
\hline Regimen & Frequency/Total $^{\mathrm{a}}(\%)$ & CR or VGPR, $\mathrm{n}(\%$ rate $)$ & OR, $\mathrm{n}(\%$ rate) \\
\hline VAD \pm VTD & $12 / 30(40)$ & $5 / 12(42)$ & $9 / 12(75)$ \\
TD & $8 / 30(27)$ & $5 / 8(62.5)$ & $6 / 8(75)$ \\
CTD & $2 / 30(7)$ & $1 / 2(50)$ & $1 / 2(50)$ \\
MP & $2 / 30(7)$ & $0 / 2(0)$ & $1 / 2(50)$ \\
VD & $2 / 30(7)$ & $1 / 2(50)$ & $1 / 2(50)$ \\
VCD & $1 / 30(3)$ & $0 / 1(0)$ & $0 / 1(0)$ \\
MPT & $1 / 30(3)$ & $1 / 1(100)$ & $1 / 1(100)$ \\
CD & $1 / 30(3)$ & $1 / 1(100)$ & $1 / 1(100)$ \\
Dexamethasone only & $1 / 30(3)$ & $\mathrm{NA}$ & $\mathrm{NA}$ \\
\hline
\end{tabular}

$\mathrm{CR}$, complete response; VGPR, very good partial response; OR, overall response; VAD, vincristine/doxorubicin/dexamethasone; VTD, bortezomib/thalidomide/dexamethasone; TD, thalidomide/dexamethasone; CTD; cyclophosphamide/thalidomide/dexamethasone; MP, melphalan/prednisolone; VD, bortezomib/dexamethasone; VCD, bortezomib/cyclophosphamide/ dexamethasone; MPT, melphalan/prednisolone/thalidomide; CD, cyclophosphamide/dexamethasone; NA, not available.

${ }^{a}$ Total indicates the number of patients with sufficient data for categorization. 
Bortezomib/dexamethasone (VD) with or without other agents (such as thalidomide, lenalidomide, doxorubicin, or cyclophosphamide) was administered in 16 patients, of whom $31 \%$ achieved CR or VGPR, $25 \%$ achieved partial response (PR), 25\% achieved stable disease, and $19 \%$ achieved PD. Only three of these patients received a bortezomib-containing regimen as a first-line treatment. Radiation therapy was performed in seven patients for the palliation of symptoms.

Among the 29 patients with relevant data, 23 (79\%) received aHSCT at any stage of treatment. Of these, 18 $(62 \%)$ received it as a part of a first-line treatment. Four patients received aHSCT more than once, either as a tandem transplantation $(\mathrm{n}=3)$ or at separate stages (n $=1)$. Before aHSCT, $36 \%$ of the patients were in CR or VGPR, and 55\% were in PR. After aHSCT, 64\% achieved CR or VGPR, and $23 \%$ achieved PR, yielding an overall
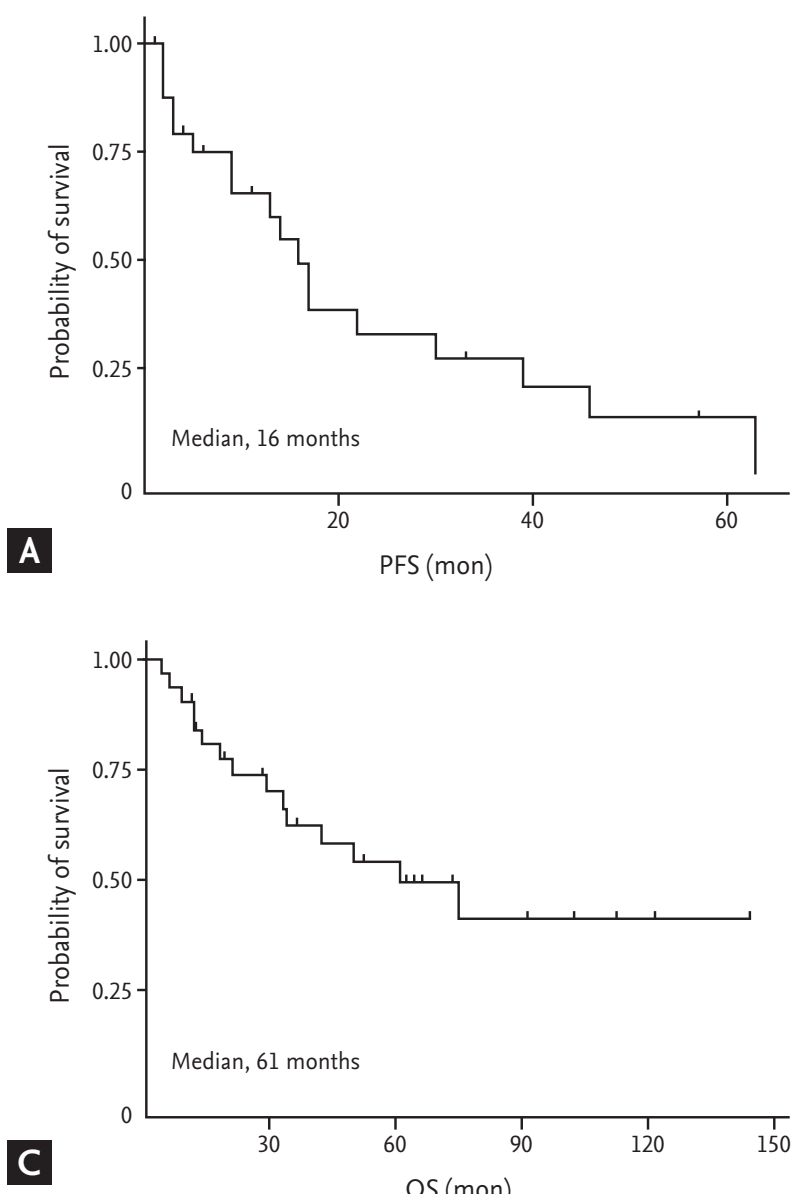

response rate of $87 \%$. Throughout the entire course of treatment, six patients did not receive aHSCT: two refused aHSCT; two were preparing for allogeneic hematopoietic stem cell transplantation (alloHSCT) because of secondary aplastic anemia and MDS; one died without having responded to chemotherapy; and one committed suicide during the induction of chemotherapy.

\section{Overall survival}

With a median follow-up duration of 64 months (interquartile range, 35 to 105) for the entire cohort, 15 out of 32 patients died. The 1-, 3-, and 5-year OS rates were $84 \%$ (95\% CI, 66\% to 93\%), 62\% (95\% CI, $42 \%$ to $77 \%$ ), and $54 \%$ (95\% CI, 34\% to $71 \%$ ), respectively. The median OS was 61 months ( $95 \%$ CI, 33 to not reached months) for the entire cohort (Fig. 1 C). No significant difference in OS was observed between subgroups divided by whether treated
B
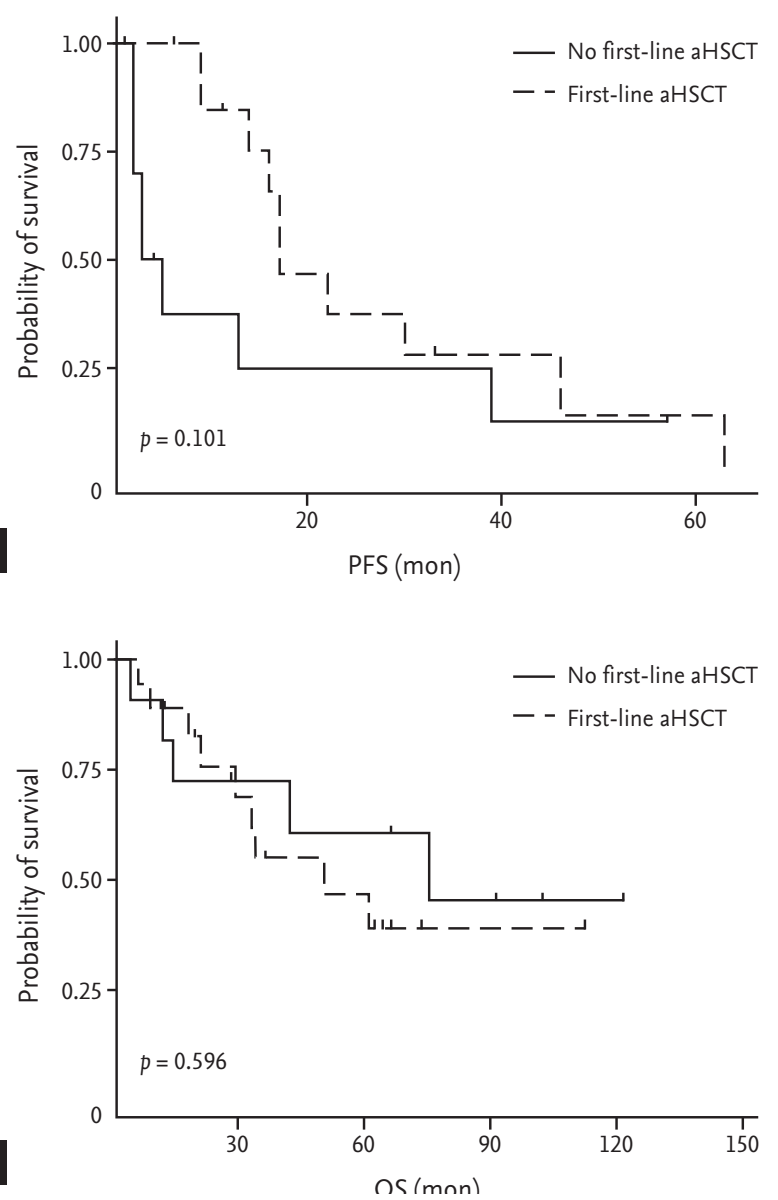

Figure 1. (A) Progression-free survival (PFS) after first-line treatment. (B) PFS after first-line treatment stratified by whether the patients received autologous hematopoietic stem cell transplantation (aHSCT) as part of the first-line treatment. (C) Overall survival (OS). (D) OS stratified by whether the patients received aHSCT as part of the first-line treatment. 
with aHSCT as the first-line treatment or not (median 50 months vs. 75 months. $p=0.596$ ) (Fig. $1 D$ ). There was a tendency toward improved OS for patients with a lower ISS stage (median 75 months [I] vs. 50 months [II] vs. 33 months [III], $p=0.363$ ), a $\kappa$ over a lambda subtype (median 75 months vs. 42 months, $p=0.161$ ), whole immunoglobulin subtype over light chain myeloma (median 75 months vs. 29 months, $p=0.219$ ), and those with biopsy-proven plasmacytoma at diagnosis (median 42 months vs. 61 months, $p=0.340$ ); though, no statistical significance was observed (Fig. 2).

The most common cause of death was myeloma progression (67\%), followed by infection (20\%). Therapy-related mortality after first-line induction chemotherapy or aHSCT, defined as death within 4 weeks after each treatment, was not observed in our cohort.
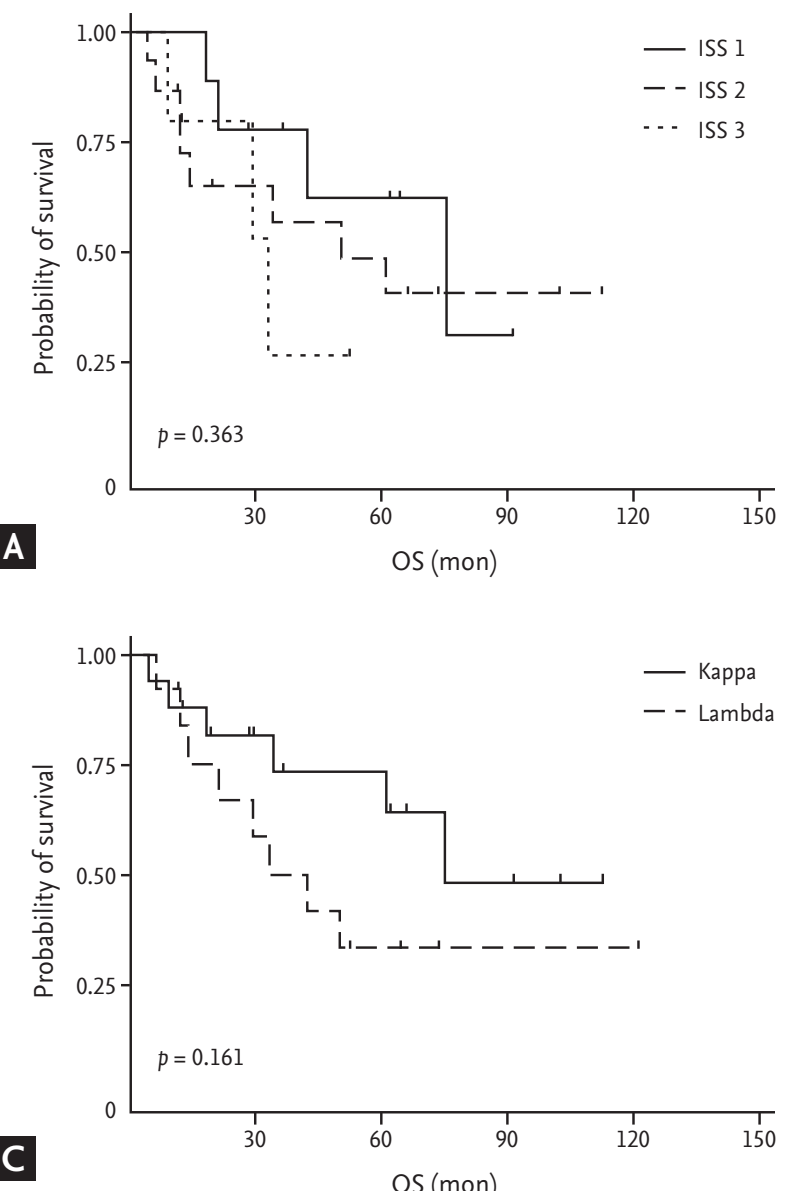

\section{DISCUSSION}

To the best of our knowledge, our study is the first case series report focusing on MM patients aged 40 years or younger at diagnosis in South Korea. In this study, we analyzed clinicopathological characteristics of extremely young MM patients to detect and accentuate any small differences in clinical features compared with the relatively well-studied population of elderly patients. Although several studies have been conducted to address the distinct clinical features of these patients in other ethnic groups, many large-scale studies used a cutoff age of 50 years $[2,4]$. Important clinical characteristics of cohorts in various previous studies being compared with our cohort are summarized in Table 4. Several presenting features in our cohort were in accordance with previous reports $[2-4,8,17]$. To be specific, the patients
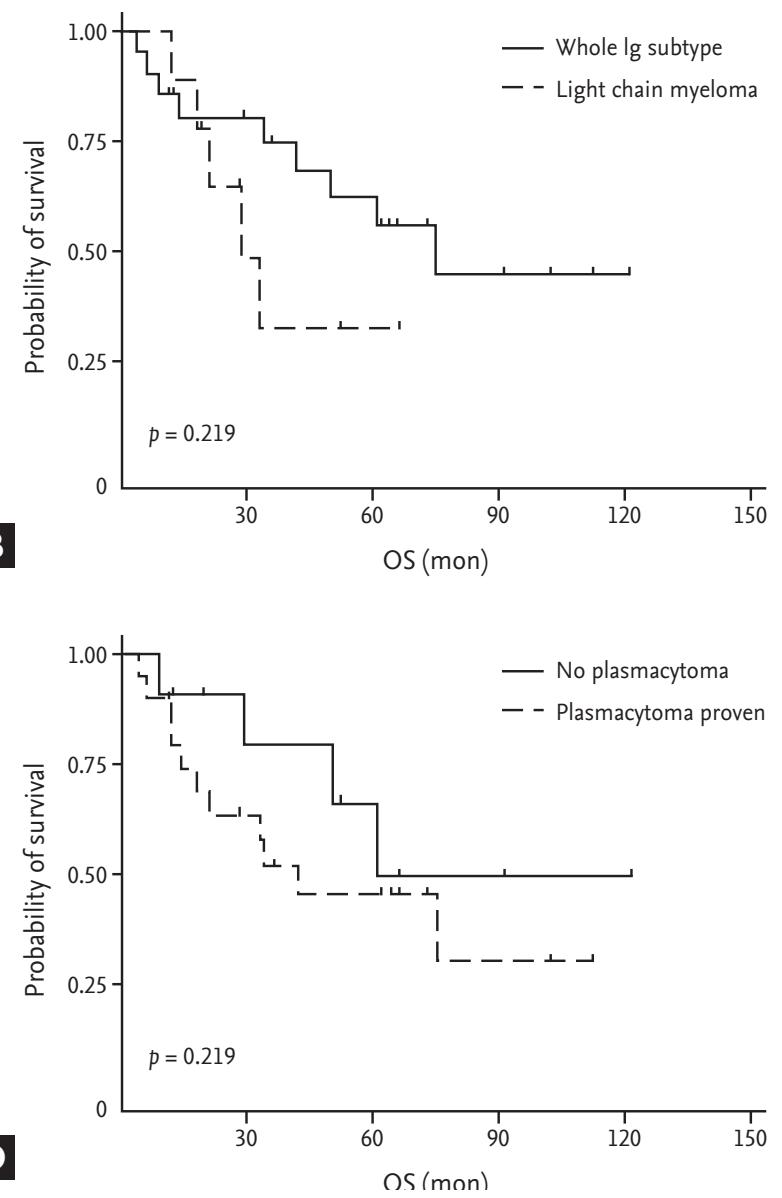

Figure 2. (A) Overall survival (OS) of subgroups divided by International Staging System (ISS) stage. (B) OS of subgroups divided into whole immunoglobulin (Ig)-secreting myeloma or light chain myeloma. (C) OS of subgroups divided by light chain subtype. (D) OS of subgroups divided by the presence of biopsy-proven plasmacytoma at diagnosis. 
were in relatively low ISS stages and had a good performance status at diagnosis compared with the historical controls of elderly MM patients $32 \%, 48 \%$, and $19 \%$ vs. $26 \%, 39 \%$, and $34 \%$ for stage I, II, and III, respectively, in patients aged 50 years or older) [4]. The proportion of lytic bone lesions involving three or more parts (45\%) was comparable to that in another large MM cohort of patients younger than 50 (48\%) [4].

However, some notable findings were revealed in our study data. First of all, a remarkably large proportion (30\%) was confirmed to have light chain myeloma, compared to $12 \%$ and $13 \%$ among patients younger than $50[2,4]$ and $12 \%$ and $21 \%$ among those aged 40 years or younger in other studies $[5,8]$. Furthermore, the BM plasma cell fraction below $30 \%$ was observed in $74 \%$ compared to $28 \%$ and $42 \%$ among similar age groups in previous studies $[4,5]$. Organ injuries indicated by anemia, hypercalcemia, azotemia, and hypoalbuminemia were observed at a low frequency compared to both of the young and old age groups in a previous large MM cohort study [4]. Most importantly, despite many favorable prognostic factors mentioned above such as low stages at diagnosis, good performance status, and a low frequency of tissue injuries, the median OS was only 61 months. This was much shorter than those observed in other two cohorts, one including patients aged 41 to 65 (longer than 80.7 months) and the other including patients aged 60 to 65 ( 89 months) $[8,11]$. Therefore, we could not find any tendency toward improved survival for young patients compared to moderately elderly patients despite previous reports suggesting that young age is a good prognostic factor $[4,11]$. This discrepancy

Table 4. Comparison of patient characteristics between several multiple myeloma cohort studies

\begin{tabular}{|c|c|c|c|c|c|c|c|c|}
\hline \multirow{2}{*}{ Characteristic } & \multirow{2}{*}{$\begin{array}{l}\text { This cohort } \\
\quad(\mathrm{n}=32)\end{array}$} & \multicolumn{2}{|c|}{ Ludwig et al. (2008) [4] } & \multicolumn{2}{|c|}{ Corso et al. (1998) [2] } & \multicolumn{2}{|c|}{ Cheema et al. (2009) [8] } & \multirow{2}{*}{$\begin{array}{l}\text { Chretien et } \\
\frac{\text { al. }(2014)[11]}{\text { Older }} \\
(\mathrm{n}=813)\end{array}$} \\
\hline & & $\begin{array}{l}\text { Younger } \\
(\mathrm{n}=1,689)\end{array}$ & $\begin{array}{c}\text { Older } \\
(\mathrm{n}=8,860)\end{array}$ & $\begin{array}{l}\text { Younger } \\
(\mathrm{n}=6 \mathrm{l})\end{array}$ & $\begin{array}{c}\text { Older } \\
(\mathrm{n}=295)\end{array}$ & $\begin{array}{l}\text { Younger } \\
(n=38)\end{array}$ & $\begin{array}{c}\text { Older } \\
(n=608)\end{array}$ & \\
\hline Male sex, \% & 59 & 61 & 57 & 52 & 57 & 61 & 60 & NA \\
\hline Age, yr, median (range) & $37(17-40)$ & $36(20-49)$ & $62(50-93)$ & $45(33-49)$ & $63(50-87)$ & $37.2(29-40)$ & $56.4(41-65)$ & NA $(60-65)$ \\
\hline \multicolumn{9}{|c|}{ 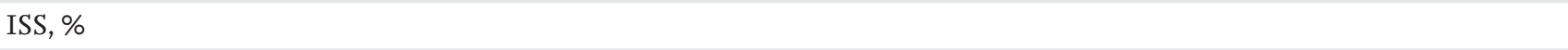 } \\
\hline $\mathrm{I}$ & 32 & 39 & 26 & 30 & 34 & 48 & NA & 30 \\
\hline II & 48 & 35 & 39 & 13 & 20 & NA & NA & 40.40 \\
\hline III & 19 & 27 & 34 & 57 & 46 & NA & NA & 29.70 \\
\hline \multicolumn{9}{|l|}{ Subtype, \% } \\
\hline Light chain only & 30 & 13 & 10 & NA & NA & 21 & NA & NA \\
\hline Immunoglobulin G & 47 & 60 & 60 & 65 & 67 & 53 & NA & NA \\
\hline Immunoglobulin A & 17 & 21 & 25 & 18 & 20 & 18 & NA & NA \\
\hline Immunoglobulin D & 7 & 3 & 3 & 1 & 1 & 8 & NA & NA \\
\hline Hemoglobin < $10 \mathrm{~g} / \mathrm{dL}, \%$ & 29 & 37 & 41 & NA & NA & NA & NA & NA \\
\hline $\mathrm{Ca}^{\mathrm{a}} 10 \mathrm{mg} / \mathrm{dL}, \%$ & 28 & 33 & 34 & NA & NA & NA & NA & NA \\
\hline $\mathrm{Cr} \geq 2 \mathrm{mg} / \mathrm{dL}, \%$ & 13 & 15 & 17 & 8 & 14 & 25 & 16 & NA \\
\hline BMPC 30\%, \% & 26 & NA & NA & NA & NA & 72 & 74 & NA \\
\hline Bone disease, \% & 87 & 79 & 78 & 43 & 34 & 76 & 74 & NA \\
\hline Upfront aHSCT, \% & 62 & 41 & 24 & NA & NA & $100^{b}$ & 31.60 & $100^{c}$ \\
\hline Median OS, mon & 61 & NA & NA & NA & NA & 81.4 & $>80.7^{\mathrm{d}}$ & 89 \\
\hline
\end{tabular}

NA, not available; ISS, International Staging System; BMPC, bone marrow plasma cell; aHSCT, autologous hematopoietic stem cell transplantation; OS, overall survival.

${ }^{\mathrm{a}}$ Corrected calcium level, calculated from measured calcium $+0.8 \times(4-$ albumin $)$.

${ }^{b}$ Upfront aHSCT was included in the inclusion criteria.

${ }^{\mathrm{c}}$ All patients were treated with 3-4 cycles of induction chemotherapy followed by 1-2 courses of high-dose melphalan.

${ }^{\mathrm{d}}$ Median OS was recorded only from the date of aHSCT and not from the date of diagnosis. 
might be partially due to the high proportion of light chain myeloma in our cohort which is associated with poor survival and relatively low proportion of patients who received upfront aHSCT (Table 4). However, considering that most previous studies included patients who had been diagnosed at least 5 to 10 years earlier than our cohort when recently developed highly effective therapeutic agents such as bortezomib, thalidomide, and lenalidomide were not available, the lack of a superior outcome in our cohort compared with these series of moderately elderly patients is notable.

A few case reports or case series have implied the existence of some unusual features of manifestation of young MM patients. For instance, a specific subgroup has been shown to manifest initially with multiple osteolytic lesions or extramedullary involvement without BM infiltration of myeloma cells. These patients seemed to have a lower tumor burden and better survival than conventional MM patients of similar ages [3,5]. Another subgroup has been characterized with multifocal liver sinusoidal infiltration of myeloma cells, presenting with fatigue, weight loss, and abdominal distension as initial symptoms [6]. In response to these findings, we separately analyzed the patients who initially had biopsyproven plasmacytoma. The low proportion of BM plasmacytosis in this subset of patients suggests that a part of this subgroup has presented with only extramedullary plasmacytoma without BM infiltration of neoplastic cells. However, these patients seemed to have short survival compared with those without plasmacytoma at diagnosis, which is not accordant with the previous reports indicating a favorable outcome of macrofocal MM $[3,5]$.

High-dose therapy (HDT) followed by aHSCT has long been considered a standard treatment for MM patients younger than 65 years of age and shown to be superior to conventional combination chemotherapy in terms of response rate, PFS, and OS [18]. Therefore, virtually all patients enrolled in this study were candidates for HDT plus aHSCT. Nevertheless, $10 \%$ of the patients were initially treated with a regimen containing melphalan, a myelotoxic agent that can compromise the BM stem cell reservoir and thus hinder stem cell harvest. One of the patients was diagnosed in 2008 and eventually received aHSCT after second-line VD, but the remaining two patients, diagnosed in 2002 and 2007 , respectively, did not receive aHSCT through their entire course of treatment. Considering that the superiority of HDT plus aHSCT over conventional therapy was demonstrated and reported in 2003 in a multicenter randomized controlled trial [18], it is unclear why these patients were initially treated with myelotoxic agents. Although a full medical history was not available for these patients, a reasonable explanation might be that their performance or comorbidity was too poor to deliver HDT at the initiation of treatment.

Our study has several limitations. First, this study was based on a small number of patients; therefore, the results should be treated with caution. Second, the source of data is a retrospective medical chart review, which made many clinical and laboratory parameters for individual patients unavailable. Third, because the national death registry data were available only for the 25 patients treated at SNUH, some patients who might have already died have been censored in the survival analysis.

Despite the limitations, a strength of our study is that a vast range of clinicopathological characteristics were addressed comprehensively for the entire cohort. The proportions of missing data were small for each analyzed variable. Although the number of studied patients is small, this study is still valuable considering the rareness of reports regarding young MM patients in Asian population. Although many laboratory parameters suggested a good prognosis in our cohort, those were not actually linked to an OS benefit compared to the moderately elderly patients in historical data [8,11]. Our study implies that an extremely young age at diagnosis does not always predict a good response to treatment. Rather, heterogeneous groups of individual patients may exist within those young MM patients. Thus, for those who are expected to show a poor response to current treatments despite their young age, the efficacy of an alternative therapeutic strategy, such as frontline alloHSCT, should be further investigated. The reason for this prognostic heterogeneity remains unclear.

\section{KEY MESSAGE}

1. In Korean multiple myeloma patients aged 40 years or younger, light chain myeloma accounted for a larger proportion while bone marrow plasmacytosis and end organ injuries were less 
prevalent than in the historical control.

2. Despite the favorable prognostic factors including low stages at diagnosis, good performance, and low frequency of tissue injuries, prognosis was poor in extremely young multiple myeloma patients.

3. Optimization of treatment strategy is urgently needed to improve outcome in this subset of patients.

\section{Conflict of interest}

No potential conflict of interest relevant to this article was reported.

\section{REFERENCES}

1. Bergsagel D. The incidence and epidemiology of plasma cell neoplasms. Stem Cells 1995;13 Suppl 2:1-9.

2. Corso A, Klersy C, Lazzarino M, Bernasconi C. Multiple myeloma in younger patients: the role of age as prognostic factor. Ann Hematol 1998;76:67-72.

3. Blade J, Kyle RA. Multiple myeloma in young patients: clinical presentation and treatment approach. Leuk Lymphoma 1998;30:493-501.

4. Ludwig H, Durie BG, Bolejack V, et al. Myeloma in patients younger than age 50 years presents with more favorable features and shows better survival: an analysis of 10549 patients from the International Myeloma Working Group. Blood 2008;111:4039-4047.

5. Dimopoulos MA, Pouli A, Anagnostopoulos A, et al. Macrofocal multiple myeloma in young patients: a distinct entity with favorable prognosis. Leuk Lymphoma 2006;47:15531556.

6. Solves P, de la Rubia J, Jarque I, et al. Liver disease as primary manifestation of multiple myeloma in a young man. Leuk Res 1999;23:403-405.

7. Hogan MC, Lee A, Solberg LA, Thome SD. Unusual presentation of multiple myeloma with unilateral visual loss and numb chin syndrome in a young adult. Am J Hema- tol 2002;70:55-59.

8. Cheema PK, Zadeh S, Kukreti V, et al. Age 40 years and under does not confer superior prognosis in patients with multiple myeloma undergoing upfront autologous stem cell transmplant. Biol Blood Marrow Transplant 2009;15:686-693.

9. Kyle RA. Prognostic factors in multiple myeloma. Stem Cells 1995;13 Suppl 2:56-63.

10. Bergsagel PL, Mateos MV, Gutierrez NC, Rajkumar SV, San Miguel JF. Improving overall survival and overcoming adverse prognosis in the treatment of cytogenetically high-risk multiple myeloma. Blood 2013;121:884-892.

11. Chretien ML, Hebraud B, Cances-Lauwers V, et al. Age is a prognostic factor even among patients with multiple myeloma younger than 66 years treated with high-dose melphalan: the IFM experience on 2316 patients. Haematologica 2014;99:1236-1238.

12. Kim K, Lee JH, Kim JS, et al. Clinical profiles of multiple myeloma in Asia-An Asian Myeloma Network study. Am J Hematol 2014;89:751-756.

13. Koh Y, Bang SM, Lee JH, et al. Low incidence of clinically apparent thromboembolism in Korean patients with multiple myeloma treated with thalidomide. Ann Hematol 2010;89:201-206.

14. Ailawadhi S, Aldoss IT, Yang D, et al. Outcome disparities in multiple myeloma: a SEER-based comparative analysis of ethnic subgroups. Br J Haematol 2012;158:91-98.

15. Rajkumar SV, Gahrton G, Bergsagel PL. Approach to the treatment of multiple myeloma: a clash of philosophies. Blood 2011;118:3205-3211.

16. Boyd KD, Ross FM, Chiecchio L, et al. A novel prognostic model in myeloma based on co-segregating adverse FISH lesions and the ISS: analysis of patients treated in the MRC Myeloma IX trial. Leukemia 2012;26:349-355.

17. Blade J, Kyle RA, Greipp PR. Presenting features and prognosis in 72 patients with multiple myeloma who were younger than 40 years. Br J Haematol 1996;93:345351.

18. Child JA, Morgan GJ, Davies FE, et al. High-dose chemotherapy with hematopoietic stem-cell rescue for multiple myeloma. N Engl J Med 2003;348:1875-1883. 